\title{
Sostenibilidad de sistemas ganaderos bovinos de alta montaña en Colombia
}

\section{Sustainability of high mountain livestock systems in Colombia}

\section{Sustentabilidade dos sistemas bovinos de pecuária de alta montanha na Colômbia}

\author{
Raúl Andrés Molina Benavides \& Hugo Sánchez Guerrero² \\ ZZootecnista, Magister en Ciencias Agrarias, Doctor en Ciencias Agrarias ${ }^{2}$ Zootecnista, \\ Especialista en Producción de Ganado Lechero, Magister en Producción Animal. \\ 1,2Departamento de Ciencia Animal, Universidad Nacional de Colombia. Palmira, Colombia. \\ 1'ramolinab@unal.edu.co, 2hsanchezg@unal.edu.co
}

\section{Resumen}

La complejidad de los sistemas agropecuarios dadas sus múltiples interacciones de elementos bióticos y abióticos, hace que estudios integrales sean importantes para un mejor entendimiento y actuación frente a éstos. Este trabajo tuvo como objetivo estudiar la sostenibilidad de 8 sistemas ganaderos alto andinos. Se encontró en promedio carga animal de 0,5 UGG, producción de leche de $4 \mathrm{~kg} / \mathrm{vaca} /$ día, intervalos de partos de 450 días y producciones de forraje de $2,45 \mathrm{t} \mathrm{MS/ha}$ con $14 \%$ PC y $60 \%$ FDN. En promedio para el bosque, el carbono orgánico del suelo fue de $33,72 \mathrm{t} / \mathrm{ha}$ y para los potreros de pastoreo de 25,29t/ha, siendo significativa la diferencia $(p \leq 0,05)$. No se encontró diferencias $(p \geq 0,05)$ entre las densidades aparentes en el suelo de los bosques nativos $\left(0,46 \mathrm{~g} / \mathrm{cm}^{3}\right)$ y los potreros $\left(0,5 \mathrm{~g} / \mathrm{cm}^{3}\right)$. Las cantidades de $\mathrm{CO}_{2}$ equivalente $\left(\mathrm{CO}_{2} \mathrm{eq}\right)$ producidas en promedio fueron de $76,12 \mathrm{t} /$ predio $(65,5 \mathrm{t}$ $\mathrm{CO}_{2}$ eq correspondientes a la producción de $\mathrm{CH}_{4}$ y 10,87 t $\mathrm{CO}_{2}$ eq a la producción de $\mathrm{N}_{2} \mathrm{O}$ ). El agua virtual requerida en promedio para los bovinos fue de $16.222,26 \mathrm{~m}^{3}$ para cubrir sus necesidades de alimento, bebida y servicios (10 años), y para producir un litro de leche requerían $1,78 \mathrm{~m}^{3}$. En el aspecto social los indicadores con menor calificación fueron servicios públicos y acceso a educación. En conclusión y sabiendo que la sostenibilidad se logra cuando hay un balance entre los componentes económico, ambiental y social; los resultados encontrados permiten afirmar que los sistemas productivos ganaderos de alta montaña incluidos en este trabajo no son sostenibles.

Palabras clave: agua virtual, carbono orgánico en el suelo, densidad aparente, gases efecto invernadero, simulación.

\section{Abstract}

The complexity of the farming systems given their multiple interactions of biotic and abiotic elements, makes integral studies important for a better understanding and action in front of them. This work was aimed at studying the sustainability of 8 high Andean livestock systems. It was found on average animal load of $0.5 \mathrm{UGG}$, milk production of 4 $\mathrm{kg} / \mathrm{cow} /$ day, calving intervals of 450 days and forage yields of $2.45 \mathrm{t} \mathrm{dm} /$ ha with $14 \% \mathrm{PC}$ and $60 \%$ NDF. On average for the forest, the organic carbon 
of the soil was $33.72 \mathrm{t} / \mathrm{ha}$ and for grazing pastures of $25.29 \mathrm{t} / \mathrm{ha}$, the difference being significant $(p \leq 0.05)$. No differences $(p \geq 0.05)$ were found between the apparent densities in the soil of native forests $\left(0,46 \mathrm{~g} / \mathrm{cm}^{3}\right)$ and pastures $\left(0.5 \mathrm{~g} / \mathrm{cm}^{3}\right)$. The equivalent amounts of $\mathrm{CO}_{2}\left(\mathrm{CO}_{2}\right.$ eq) produced on average were $76.12 \mathrm{t} /$ site $\left(65.5 \mathrm{t} \mathrm{CO}_{2}\right.$ eq corresponding to the production of $\mathrm{CH} 4$ and $10.87 \mathrm{~T} \mathrm{CO}_{2}$ eq to the production of $\mathrm{N}_{2} \mathrm{O}$ ). The virtual water required on average for bovine animals was 16,222.26 $\mathrm{m}^{3}$ to meet their food, beverage and service needs (10 years), and to produce a litre of milk, they needed $1.78 \mathrm{~m}^{3}$. In the social aspect the indicators with lower qualification were public services and access to education. In conclusion and knowing that sustainability is achieved when there is a balance between the economic, environmental and social components; The results found make it possible to say that the high-mountain livestock production systems included in this work are not sustainable.

Key-words: virtual water, organic carbon in the soil, apparent density, greenhouse gases, simulation

\section{Resumo}

A complexidade dos sistemas agrícolas, considerando suas múltiplas interações de elementos bióticos e abióticos, faz estudos integrais importantes para uma melhor compreensão e ação contra eles. Este estudo teve como objetivo estudar a sustentabilidade de 8 sistemas de gado andino alto. A carga animal média foi de 0,5 UGG, produção de leite $4 \mathrm{~kg}$ / vaca / dia, intervalos de parto de 450 dias e rendimentos de forragem de $2,45 \mathrm{t}$ DM / ha com $14 \%$ de CP e $60 \%$ de FDN. Em média, para a floresta, o carbono orgânico do solo foi de 33,72 t / ha e para pastagens de 25,29 $t /$ ha, sendo a diferença significante $(p \leq 0,05)$. Não houve diferenças $(p \geq 0,05)$ entre as densidades aparentes do solo em florestas nativas $\left(0,46 \mathrm{~g} / \mathrm{cm}^{3}\right)$ e pastagens $\left(0,5 \mathrm{~g} / \mathrm{cm}^{3}\right)$. As quantidades de $\mathrm{CO}_{2}$ equivalente $\left(\mathrm{CO}_{2} \mathrm{eq}\right)$ produziram em média $76,12 \mathrm{t} /$ planta (65,5 t CO $\mathrm{CO}_{2}$ eq correspondente à produção de $\mathrm{CH} 4 \mathrm{e}$ 10,87 t $\mathrm{CO}_{2}$ eq à produção de N2O). A água virtual requerida em média para o gado foi de $16.222,26 \mathrm{~m}^{3}$ para cobrir suas necessidades de alimentos, bebidas e serviços (10 anos) e produzir um litro de leite exigiu $1,78 \mathrm{~m}^{3}$. No aspecto social, os indicadores com menor qualificação foram os serviços públicos e o acesso à educação. Em conclusão e sabendo que a sustentabilidade é alcançada quando há um equilíbrio entre os componentes econômicos, ambientais e sociais; os resultados encontrados permitem afirmar que os sistemas de produção de gado de alta montanha incluídos neste trabalho não são sustentáveis.

Palavras chave: água virtual, carbono orgânico no solo, densidade aparente, gases de efeito estufa, simulação.

\section{Introducción}

La actividad ganadera en los trópicos domina la escena global en cuanto al número de animales, producción total y número de beneficiarios en comparación con la producción pecuaria del mundo occidental (Oosting et al., 2014).

En años recientes, la actividad ganadera en el trópico ha tenido más auge en los discursos científicos y sociales debido a tres factores principalmente: primero, la demanda de carne y leche en los paises en desarrollo se ha incrementado y lo seguirá haciendo (FAO, 2009); segundo, las emisiones de gases de efecto invernadero atribuidas a la producción ganadera es alta (FAO, 2006; de Vries \& de Boer, 2009; Gerber et al., 2011; Herrero et al., 2011) y tercero, muchos de los ganaderos en el trópico son pobres, por lo tanto el mejoramiento de la ganadería conduce a menguar esa pobreza (World Bank, 2007, 2009; Herrero et al., 2013).

En Colombia, la ganadería representa una de las actividades más importantes, no solo por sus aportes directos en la alimentación -leche y carne-, sino por todas aquellas actividades derivadas a partir de 
la transformación de sus productos. Esta actividad se desarrolla en las cinco grandes regiones biogeográficas, (Andina, Amazonia, Caribe, Orinoquia y Pacífica) del país y sobre todas las altitudes. Según Galindo \& Muergueitio (2007), las actividades ganaderas se han realizado tradicionalmente transformando los bosques alto andinos y páramos a través de sistemas de producción que tienden a ser de lechería en las zonas de altiplanos y páramos bajos (2.000 - $3.200 \mathrm{msnm})$; de doble propósito -carne y leche- o cría para laderas de alta montaña y páramos (>3.500 msnm).

Colombia posee un hato bovino cercano a los 23 millones de cabezas ubicados en 39.2 millones de hectáreas, presentando una carga de 0.6 animales/hectárea (Fedegan, 2013a), hecho que clasifíca la actividad de tipo extensivo, en la cual se usan pastos de especies nativas y naturalizadas, como principal recurso alimenticio, de bajo valor nutritivo. Esta carga animal no ha variado significativamente en los últimos veinte años, lo que revela la pobre transformación tecnológica del sector ganadero (PNUD, 2011).

En la actualidad, la necesidad de sistemas ganaderos que sean economicamente viables, ambientalmente responsables y socialmente justos, es decir sistemas productivos sostenibles, es inmediata (Cooprider et al., 2011). Por lo tanto, el futuro de la ganadería está en la capacidad de este logro, es decir la actividad ganadera moderna y posmoderna tiene como reto principal, modificar sus relaciones de eficiencia y eficacia con el estado de los elementos naturales que usa.

Sin embargo, la principal limitante para plantear estrategias de reconversión hacia una ganadería sostenible es el desconocimiento sobre las técnicas usadas por los propietarios y los beneficios financieros que éstos obtienen de su actividad. Debido a esto, es necesario estudiar de forma integral la actividad productiva ganadera que se desarrolla en el país, con el fin de encontrar estrategias que mejoren las condiciones de vida de las familias en esos territorios, sin detrimento de los recursos naturales.
Por lo anteriormente expuesto, el objetivo de la investigación fue estudiar la sostenibilidad de los sistemas ganaderos localizados en el Parque Nacional Natural de las Hermosas y su zona de influencia mediante la caracterización, mediciones de campo, uso de simuladores y herramientas de monitoreo en los predios trabajados.

\section{Materiales y métodos}

\section{Área de estudio}

El estudio se realizó en ocho predios localizados en el sector de La Nevera, ubicado en las tierras altas de la vertiente occidental de la cordillera Central, en jurisdicción del municipio de Palmira, Valle, Colombia, en el límite de vertiente entre las cuencas de los ríos Amaime -Toche- y Nima. Los predios estaban entre los 2700 y 3600 msnm, con temperaturas promedio de $13^{\circ} \mathrm{C}$, precipitaciones anuales entre los 1600 y $1800 \mathrm{~mm}$ y humedad relativa mayor al $80 \%$. (Ospina \& Molina, 2009)

\section{Caracterización y mediciones de campo en los predios}

La caracterización de los sistemas productivos se hizo mediante encuestas estructuradas con los propietarios, análisis bromatológicos de los forrajes, mediciones de campo como la disponibilidad de forraje (método de disponibilidad por frecuencia; Franco et al, 2006), determinaciones del Carbono Orgánico en el Suelo (COS) y Densidad aparente (Da) (método de Walkley and Black y metodo del anillo, (IGAC, 2007), respectivamente). Para la determinación del COS y $\mathrm{Da}$, se tomaron 192 muestras de suelo en los ocho predios, a dos rangos de profundidad (0-5 y $5-10 \mathrm{~cm}$ ), en cuatro sitios de muestreo -una en bosque nativo y tres en potreros destinados para el pastoreo del ganado-, con tres repeticiones por sitio de muestreo. Se realizó análisis de varianza, utilizando el diseño de parcelas divididas, con el objetivo de observar cual era el comportamiento de las variables a evaluar, carbono orgánico t/ha y densidad aparente $\mathrm{gr} / \mathrm{cm}^{3}$, dichas variables fueron evaluadas por la metodología de diferencia de medias de Duncan (SAS, 2009). 


\section{Simulaciones}

Con el fin de estimar el comportamiento productivo de los animales basado en la alimentación recibida y simular posibles estrategias de alimentación económica y ambientalmente viables, se trabajo con el software LIFE-SIM (León Velarde et al., 2006). La simulación se hizo para un animal en el periodo de un año. Se plantearon tres escenarios diferentes: 1 línea base (datos reales); 2 Fertilización (Fert) de los potreros y mejores parámetros productivos y reproductivos (MPR), 3 Fert + MPR + Suplementación.

Para determinar la cantidad de gases de efecto invernadero (GEI) t $\mathrm{CO}_{2}$ equivalente/t de solidos de leche, emitidos en cada uno de los 8 predios, se trabajó con el simulador DGAS (Dairy Greenhouse Gas abatement strategy Calculator - farmer, versión 2009), en el cual se introdujeron datos correspondientes a lo encontrado en la caracterización de cada sistema productivo. Por cada predio se simularon 5 escenarios diferentes con el fin de comparar la producción de GEI de la línea base. Los escenarios fueron los siguientes: 1 Línea base, 2 Suplementación + Mejores Parámetros Productivos (MPP), 3 Fertilización (Fert) + MPP, 4 Suplementación + Fert + MPP y 5 Línea base sin área en bosque. También se midió los kg $\mathrm{CO}_{2}$ e emitidos en el predio por kg leche corregida producida en este.

Para estimar la cantidad de agua consumida -agua virtual- por un bovino desde su nacimiento hasta el sacrificio, 0 a 10 años, se siguió la metodología propuesta por Chapagain \& Hoekstra, 2003, iniciando con la ecuación 1.

$\mathrm{CAV}=\mathrm{CAVa}+\mathrm{CAVb}+\mathrm{CAVs}$

CAV: Cantidad de agua virtual, a: alimento, b: bebida y s: servicios

En donde la cantidad de agua consumida es la sumatoria del agua que el animal consume en los alimentos que ingiere, el agua que bebe y el agua que utiliza para sus servicios.

\section{Herramienta de monitoreo}

Por último, la parte social de estos predios se trabajó con la ayuda de la herramienta de monitoreo técnico económico para una ganadería sostenible (HMTEGS) (Cipav, 2009). Los indicadores trabajados fueron acceso a la educación, vivienda, acceso a la salud, vinculación a servicios de salud, seguridad alimentaria, servicios públicos e ingreso neto mensual. Se calificó en una escala de 1 a 5, donde lo deseado es que en cada indicador el valor encontrado sea cinco.

\section{Resultados y discusión}

\section{Caracterización y mediciones de campo en los predios}

La actividad productiva principal en el área de estudio es la ganadería, siendo el modelo doble propósito el predominante. El área de las fincas estuvo entre 27 y 600 ha. De esta área, en promedio el $50,62 \% \pm 20,8$ son potreros destinados al pastoreo, lo restante son bosques. La carga animal promedio en estos ocho predios fue de 0,52 $\pm 0,38 \mathrm{UGG} / \mathrm{ha}$, lo que conlleva a asumir que la ganadería realizada es de tipo extensivo. Los valores de carga animal encontrados en este trabajo, concuerdan con los rangos, de acuerdo al nivel de intensificaión, presentados por Gómez \& Rueda (2011). El número total de animales por predio tuvo una relación directa con el área de la finca. El promedio de animales para los predios visitados fue de 61, encontrando fincas con 31 animales y la de mayor población con 116. El número promedio de animales en ordeño fue de 16.

Los parámetros de producción de leche indicaron que la producción promedio por vaca/día en estos predios fue de $4 \pm 1$ L. Según Corpoica (2006), la producción promedio de leche diaria en ganado doble propósito en Colombia es de $3 \mathrm{~L}$. En cuanto a parámetros reproductivos, la edad promedio al primer parto estuvo en $41,62 \pm 6,67$ meses. El intervalo entre partos y la tasa de natalidad promedio que se encontraron fue de 450 días y $81 \%$, respectivamente. Según Fedegan (2013b), la tasa de natalidad para ganadería doble propósito estaba entre 
$50-53 \%$ y el intervalo entre partos era de 680 días en promedio. Vale la pena recalcar que los valores publicados por Fedegan incluyen datos de ganaderías doble propósitos ubicadas en zonas secas y con poca dispobibildad forrajera.

En cuanto a la disponibilidad de forraje, en la época seca se encontraron producciones promedio de forraje verde de $8,5 \pm 4,8 \mathrm{t} / \mathrm{ha}$, equivalentes a $2,1 \pm 1,3$ $\mathrm{t} /$ ha de materia seca. Para esta época de muestreo los forrajes tuvieron en promedio 13,96 $\pm 3,68 \%$ PC y $56,3 \pm 3,73$ FDN. Para la época de lluvia, las producciones de forraje verde fueron de 14,1 $\pm 8,06$ t/ha, equivalentes a 2,8 $\pm 1,46 \mathrm{t} / \mathrm{ha}$ de MS. Para este periodo los forrajes presentaron en promedio PC de $14,27 \pm 4,58 \%$ y FDN de $64,88 \pm 5,53 \%$. Sarria et al, 2008, encontró en rio Negro Antioquia, producciones de 1,8 t MS/ha con $22,6 \%$ PC y $74,9 \%$ FDN. Los contenidos bajos de PC encontrados en este estudio podrían atribuirse a los periodos de descanso que se manejan en esta zona (entre 70 y 80 días), lo que aumentaría los carbohidratos estructurales. Según Peters (2008), las condiciones ambientales durante el crecimiento de la planta afectan el contenido de FDN, lo que explica su variación entre las épocas de muestreo. Durante la época seca se dieron valores altos de radiación y brillo solar, por lo tanto, el aumento en la intensidad de la luz favorece los procesos de síntesis y acumulación de carbohidratos solubles en la planta, mostrando un comportamiento inverso con el resto de los constituyentes solubles y estructurales, siempre que otros factores no sean limitantes. Esta puede ser una de las razones por las que en este trabajo se dio un mayor contenido de FDN en la época lluviosa -menor radiación y brillo solar- en comparación con la época seca.

Los resultados del contenido de carbono orgánico en el suelo $-10 \mathrm{~cm}$ profundidad- que se obtuvieron en este estudio, mostraron en promedio para los bosques cantidades de 33,72 t/ha, con predios que presentaban $18,78 \mathrm{t} /$ ha y otros con $46,93 \mathrm{t} / \mathrm{ha}$. Trabajando con la cantidad promedio de 33,72 t/ ha y expresándola en $\mathrm{CO}_{2}$ eq, se obtuvo que estos bosques almacenaban en promedio 123,75 t $\mathrm{CO}_{2}$ eq. Para los potreros destinados al pastoreo del ganado, en promedio el COS fue de 25,29 t/ha, con predios que tenían 17,7 t/ha y otros con $37,93 \mathrm{t} / \mathrm{ha}$. En los potreros se encontró que el almacenamiento era de 92,81 $\mathrm{tCO}_{2}$ eq. Al realizar el ANOVA y la posterior prueba de medias de DUNCAN, se encontró que hubo diferencias altamente significativas entre los sitios de muestreo (bosque vs potrero) y también entre las profundidades en las que se tomaron las muestras, $0-5 \mathrm{~cm}$ y $5-10$ $\mathrm{cm}$. Esto podría atribuirse a los diferentes tipos de uso del suelo, uno destinado a la conservación y el otro al pastoreo de los animales, y en cuanto a las diferencias entre las profundidades podría deberse a que los contenidos de materia orgánica fueron mayores en los primeros $5 \mathrm{~cm}$. Para los resultados obtenidos de densidad aparente, los bosques mostraron en promedio $0,46 \mathrm{~g} / \mathrm{cm}^{3}$ y para los potreros $0,5 \mathrm{~g} / \mathrm{cm}^{3}$. Al realizar el ANOVA y la posterior prueba de medias de DUNCAN, se encontró que no hubo diferencias significativas entre los sitios de muestreo (bosque vs potrero) y también entre las profundidades en las que se tomaron las muestras, $0-5 \mathrm{~cm}$ y $5-10 \mathrm{~cm}$. Esto podría atribuirse a que la ganadería que se practica en estos predios presenta una carga animal baja, 0,5 UGG/ha, y a que las cantidades de materia orgánica en estos suelos son altas.

\section{Simulaciones}

Al realizar las simulaciones con el LIFE-SIM, para el escenario 1, la producción de leche fue de 778 $\mathrm{kg}$, el margen bruto fue de $\$ 474.807$ y la producción de metano por $\mathrm{kg}$ de leche producida fue de 0,11; para el escenario 2, la producción de leche fue de $1316 \mathrm{~kg}$, el margen bruto fue de $\$ 886.172$ y la producción de metano por $\mathrm{kg}$ de leche producida fue de 0,07 y para el tercer escenario la producción de leche fue de $1782 \mathrm{~kg}$, el margen bruto fue de $\$ 260.504$ y la producción de metano por $\mathrm{kg}$ de leche producida fue de 0,06. Se pudo observar que al implementar la estrategia donde se adicionaba el suplemento alimenticio, la viabilidad económica no era la deseada para el productor. Según Molina et al. (2017), entre más productivo sea el animal, mayor será la dilución del gas en el producto, por lo tanto su huella de carbono será menor. 
Al simular las producciones de $\mathrm{CO}_{2}$ equivalentes, se encontró en promedio para estos predios -1810 t $\mathrm{CO}_{2}$ eq, este valor fue negativo debido a que el área destinada a la conservación -bosque- en cada predio, tenía mayor capacidad de captura de $\mathrm{CO}_{2}$ que la capacidad productiva del mismo. Al realizar la simulación asumiendo que en los predios no había bosque, las producciones en promedio fueron de $76,37 \mathrm{tCO}_{2}$ eq $\left(65,5 \mathrm{t} \mathrm{CO}_{2}\right.$ eq correspondientes a la producción de $\mathrm{CH}_{4}$ y 10,87 t $\mathrm{CO}_{2}$ eq a la producción de $\mathrm{N}_{2} \mathrm{O}$ ). Según Mora (2010), en fincas doble propósito de Costa Rica, las producciones anuales de $\mathrm{CO}_{2}$ eq estaban en 66,5 t. La menor cantidad de $\mathrm{CO}_{2}$ eq emitidos por los predios costarricenses, podría atribuirse a la mejor calidad de los forrajes ofrecidos y a una posible suplementación, lo que ocasionaría una disminución en las emisiones de metano producido por los procesos de fermentación ruminal.
En cuanto a la cantidad de agua virtual requerida para cada animal desde su nacimiento hasta el sacrificio estuvo en $16.222 \mathrm{~m}^{3} /$ animal $\left( \pm 36.049 \mathrm{~m}^{3} / \mathrm{t}\right)$, lo cual estuvo por debajo de lo reportado por Chapagain \& Hoekstra (2003) para ganado de leche en Colombia $141.520 \mathrm{~m}^{3} / \mathrm{t}$. En cuanto a la cantidad de agua requerida para producir un $\mathrm{kg}$ de leche, estuvo en $1,78 \mathrm{~m}^{3} / \mathrm{kg}$ leche, lo cual estuvo por encima del promedio mundial según Chapagain \& Hoekstra (2008) que era de $1 \mathrm{~m}^{3} / \mathrm{kg}$ leche. Molina et al. (2016), estimó consumos de $12.804 \mathrm{~m}^{3} / \mathrm{animal}$-desde el nacimiento hasta su sacrificio- y $2,17 \mathrm{~m}^{3} / \mathrm{kg}$ leche producida.

\section{Herramienta de monitoreo}

Por último, en la Tabla 1 se muestran los valores reportados por cada indicador para cada predio, utilizando la herramienta de monitoreo técnico económico para una ganadería sostenible (HMTEGS).

Tabla 1. Calificación indicadores sociales por predio utilizando la HMTEGS.

\begin{tabular}{|l|c|c|c|c|c|c|c|c|c}
\hline \multicolumn{1}{c}{ Indicadores } & P1 $^{*}$ & P2 & P3 & P4 & P5 & P6 & P7 & P8 & Valor total/indicador \\
\hline Vivienda & 3 & 3 & 4 & 1 & 3 & 3 & 3 & 3 & 23 \\
\hline Acceso eduacación & 1 & 1 & 1 & 1 & 1 & 1 & 1 & 1 & 8 \\
\hline Acceso salud & 2 & 2 & 2 & 2 & 2 & 2 & 2 & 2 & 16 \\
\hline Vinculación servicios salud & 3 & 2 & 5 & 2 & 5 & 3 & 5 & 3 & 28 \\
\hline Servicios públicos & 1 & 1 & 1 & 1 & 1 & 1 & 1 & 1 & 8 \\
\hline Seguridad alimentaria & 1 & 1 & 1 & 1 & 3 & 1 & 1 & 1 & 10 \\
\hline Ingreso neto mensual & 2 & 2 & 2 & 2 & 2 & 2 & 2 & 2 & 16 \\
\hline Valor total/predio & 13 & 12 & 16 & 10 & 17 & 13 & 15 & 13 & \\
\hline
\end{tabular}

${ }^{\star} \mathrm{P}=$ Predio.

El valor total por predio indicó el estado en que se encontraba cada uno de éstos, a medida que el valor era mayor, significaba que éste se encontraba en mejores condiciones. El valor total por indicador mostró cuál de éstos era el más ausente en la zona, a medida que la calificación era menor, ese aspecto social era en el que más había falencias.
El indicador vivienda, mostró que en seis de los ocho predios concordaron que el estado de ésta era regular, un predio la calificó como muy mala y otro como terminada, muy buena. Para el indicador acceso a la educación, todos los predios concordaron en que no hay acceso a programas de educación, capacitación y acompañamiento "asistencia 
técnica". Para el indicador acceso a la salud, todos los predios concordaron en que había un puesto de salud en el sector pero sin personal y mal equipado. Para el indicador vinculación a servicios de salud), hubo dos predios en los que solo algunos trabajadores estaban inscritos al SISBEN, tres predios dijeron que todos sus trabajadores estaban inscritos al SISBEN y tres predios pagaban seguridad social para todos sus trabajadores. Para el indicador servicios públicos, todos los predios coincidieron en que no poseían servicio de energía eléctrica ni agua potable. Para el indicador seguridad alimentaria, solo un predio de los ocho trabajados poseía una huerta casera, la cual los proveía de alimentos para el consumo, pero no en mucha cantidad ni calidad; este problema de inseguridad alimentaria conlleva a que todo el alimento para el consumo humano debe ser comprado en la ciudad, ocasionando que gran parte del dinero que ingresa tiene que ser utilizado en la compra de alimentos, que hubieran podido ser producidos en el mismo predio. Para el indicador ingreso neto mensual, todos los predios respondieron en que sus ingresos eran menores a cuatro y superiores o iguales a un salario mínimo.

\section{Conclusiones}

El uso de indicadores económicos, ambientales y sociales, integrados en un mismo estudio, permite tener una mejor comprensión sobre el comportamiento de los sistemas productivos.

El contenido de COS t/ha encontrado en los bosques y potreros, indican que los suelos de esta zona son reservorios de $\mathrm{CO}_{2}$ importantes, que si no son manejados adecuadamente podrían convertirse en una fuente de emisión de este gas a la atmósfera. Adicionalmente, las áreas destinadas a la conservación -bosques- en estos sistemas ganaderos, permiten contrarrestar las emisiones de $\mathrm{CO}_{2}$ eq producidas por los mismos.

Los simuladores LIFE-SIM, DGAS y la HMTEGS son herramientas útiles, de fácil manejo, ya que la información que requieren se puede obtener a nivel de campo, los resultados que arrojan son similares a lo encontrado en los predios, permitiendo tomar mejores decisiones a la hora de implementar estrategias amigables ambiental y económicamente.

Sistemas productivos intensivos en el uso de los recursos naturales: sol, agua, suelo, planta y animales, acompañados de su preservación, permitirán satisfacer las necesidades de alimentos y servicios ambientales de una población humana creciente.

De acuerdo a la investigación realizada y sabiendo que para que un sistema sea sostenible debe presentar un balance en sus componentes económico, ambiental y social; los resultados encontrados permiten afirmar que los sistemas productivos ganaderos de alta montaña incluidos en este trabajo no son sostenibles.

\section{Literatura citada}

1. Chapagain, A. K., \& Hoekstra, A. Y. (2003). Virtual water flows between nations in relation to trade in livestock and livestock products. Value of water research report series No. 13. UNESCO-IHE Institute for water education. Recuperado de: http://waterfootprint.org/ media/downloads/Report13.pdf

2. Chapagain, A. K. \& Hoekstra, A. Y. (2008). Globalization of water: Sharing the planet's freshwater resources. Blackwell Publishing, Oxford, UK. 220p.

3. CIPAV. (2009). Herramienta de monitoreo técnico económico para una ganadería sostenible. Recuperado de: http://www.cipav.org.co/index.php?option=com wrapper\&itemid $=252$.

4. Corpoica. (2006). Revista Corpoica: Ciencia y tecnología agropecuaria. Recuperado de: http://www.corpoica.org.co/SitioWeb/Revistas/Revistas.asp

5. Cooprider, K. L., Mitloehner, F. M., Famula, T. R., Kebreab, E., Zhao, Y., A. L. Van Eenennaam. (2011). Feedlot efficiency implications on greenhouse gas emissions and sustainability. Journal of Animal Science 89(8), 2643-2656.

6. De Vries, M. \& de Boer, I. (2009). Comparing environmental impacts of livestock products: a review of life cycle assessments. Livestock Science 128, 1-11.

7. DGAS. (2009). Dairy Green House gas Abatement Strategy Calculator. Farmer version User Manual-Version 1.2. Australia. http://www.piccc.org.au/resource/360

8. FAO. (2006). Livestock's long shadow. Environmental issues and options. Food and Agriculture Organization, Rome, Italy. http://www.fao.org/docrep/010/a0701e/ a0701e00.HTM

9. FAO. (2009). The state of food and agriculture - livestock in the balance. Food and Agriculture Organization, Rome, Italy. 
10. Fedegan - Federación Colombiana de Ganaderos Fondo Nacional del Ganado (2013a). Análisis del inventario ganadero Colombiano: Comportamiento y variables explicativas. $21 \mathrm{p}$.

11. Fedegan - Federación Colombiana de Ganaderos Fondo Nacional del Ganado (2013b.). Foro empresarización y competitividad ganadera: Costos y los indicadores de productividad en la ganadería Colombiana.

12. Franco, L. H.; Calero, D. \& Duran, C. V. (2006). Manejo y utilización de forrajes tropicales multipropósito. Convenio 0620 - 1. Universidad Nacional de Colombia. Sede Palmira. Gobernación del Valle del Cauca - Secretaria de agricultura y pesca.

13. Galindo. W. \& Murgueitio E. (2007). Reducción ganadera de los páramos, una contribución a la adaptación al cambio climático de la alta montaña.

14. Gerber, P., Vellinga, T., Opio, C., \& Steinfeld, H. (2011). Productivity gains and greenhouse gas emissions intensity in dairy systems. Livestock Science 139, 100 -109 .

15. Gómez, J. \& Rueda, R. (2011). Productividad del sector ganadero bovino en Colombia durante los años 2000 a 2009. (Trabajo de grado). Colegio Mayor Nuestra Señora del Rosario. Bogotá D.C. 89p.

16. Herrero, M., Gerber, P., Vellinga, T., Garnett, T., Leip, A., Opio, C., Westhoek, H., Thornton, P., Olesen, J., Hutchings, N., Montgomery, H., Soussana, J., Steinfeld, H. \& McAllister, T. (2011). Livestock and greenhouse gas emissions: the importance of getting the numbers right. Animal Feed Science and Technology.166-167, 779-782.

17. Herrero, M., Grace, D., Njuki, J., Johnson, N., Enahoro, D., Silvestri, S. \& Rufino, M. (2013). The roles of livestock in developing countries. Animal 7, 3-18.

18. IGAC - Instituto Geográfico Agustín Codazzi (2007). Manual de métodos analíticos del laboratorio de suelos, Bogotá, D.C: IGAC, 20 p. Recuperado de: http://www. igac.gov.co/wps/wcm/connect/dd516280464b0a$a b 8 a 70 c b 525 e 257 f 7 f / L I S T A D O+D E+M E T O D O S+$ $E M P L E A D O S+E N+E L+L A B O R A T O R I O+N A C \% 20$ IONAL+DE+SUELOS.pdf?MOD=AJPERES

19. León Velarde C., Quiroz R. A., Cañas R.; Osorio J., Guerrero J., \& Pezo, D. (2006). LIFE SIM: Livestock feeding strategies simulation models. Natural Resources Management Division. CIP (International Potato Center), Lima, Peru. (Working Paper no. 2006-1). 37p.

20. Molina, R., Sánchez, H., Uribe, J., \& Atzori, A. 2016. Efecto de la edad al primer parto y los días abiertos en un bovino doble propósito sobre la huella hídrica y de carbono. Revista de Investigación Agraria y Ambiental, 7 (2), 107-119.

21. Molina, R., Sánchez, H., Campos, R., Atzori, A. \& Morales, J. (2017). Dynamic estimation of greenhouse gas emissions from bovine livestock of Valle del Cauca, Colombia. Acta Agron. 66 (3), 422-429.

22. Mora, V. (2010). Balance de gases de efecto invernadero y la carbono neutralidad en sistemas agrosilvopastoriles de Costa Rica. Presentado en el VI congreso internacional de agroforesteria para la producción pecuaria. Ministerio de agricultura y ganadería - Costa Rica.

23. Oosting, S., Udo, H. \& Viets, T. (2014). Development of livestock production in the tropics: farm and farmers' perspectives. Animal. 1-11.

24. Ospina, G. \& Molina, R. (2009). Diagnóstico y caracterización de los sistemas productivos ganaderos del Parque Nacional Natural Las Hermosas y su zona de influencia en el sector de Palmira y planeación de modelos pilotos productivos a implementar en los predios caracterizados, enfocados hacia una propuesta de ordenamiento territorial que cumpla una función amortiguadora. Convenio no. 054 de 2008. Corporación Autónoma Regional del Valle del Cauca y Unidad de Parques Nacionales Naturales de Colombia. pp 121.

25. Peters, K. (2008). Valor nutricional y disponibilidad de biomasa del pasto kikuyo (Pennisetum clandestinum) en sistemas d pastoreo basados en la edad fenológica de la planta. (Tesis). Universidad de Costa Rica.

26. PNUD - Programa de las Naciones Unidas para el desarrollo en Colombia (2011). Colombia rural. Razones para la esperanza. Informe Nacional de Desarrollo Humano 2011. Bogotá: INDH PNUD. 443p.

27. Sarria, P.; Builes, A.; Restrepo, C. \& Murgueitio, E. (2008). Evaluación de la producción y calidad de kikuyo Pennisetum clandestinum asociado con árboles de aliso Alnus acuminata en los Andes Centrales, Antioquia. En el libro: Ganadería del futuro (investigación para el desarrollo). Capitulo 17.

28. World Bank. (2007). World development report 2008 : agriculture for development World Bank, Washington, DC. Recuperado de: http://bit.ly/2heWjsj

29. World Bank. (2009). Awakening Africa's sleeping giant: prospects for comercial agriculture in the Guinea savannah zone and beyond World Bank, Washington, DC. Recuperado de: http://siteresources.worldbank. org/ INTARD/Resources/sleeping_giant.pdf
Conflicto de Intereses

Los autores declaran no tener ningún conflicto de intereses

Recibido: Febrero 28 de 2017 Aceptado: Abril 07 de 2017 